\title{
Sur les courbes de convergence des séries de polynomes à une variable complexe et leur application à la détermination des fonctions holomorphes dans des domaines dounés.
}

\author{
par N. Abramesco (Cluj-Roumanie).
}

\section{INTRODUCTION}

Les séries de polynomes à une variable complexe, $\Sigma A_{n} P_{n}(x), \Sigma \frac{A_{n}}{P_{n}(x)}$, apparaissent comme une généralisation des séries $\Sigma A_{n} x^{n}, \Sigma \frac{A_{n}}{\mathfrak{x}^{n}}$. En com. mençant par les séries $\Sigma A_{n} P_{n}(x)$, on peut faire l'étude de ces séries à deux points de vue. Premièrement, étant donnée une fonction $f(x)$, régulière dans un domaine limité par la courbe $(C)$, tronver le développement de $f(x)$ en série de polynomes, $f(x)=\Sigma A_{n} P_{n}(x)$, valable seulement à l'intérieur de la courbe $(C)$. Ce problème a été résolu par M. FABER $\left({ }^{1}\right)$, qui a montré que les polynomes $P_{n}(x)$ dépendent seulement de contour $(C)$ et les coefficients $A_{n}$ dépendent de la courbe $(C)$ et de la fonction $f(x)$.

Un autre point de vue de l'étude des séries de polynomes $\Sigma A_{n} P_{n}(x)$ est le suivant. On donne les polynomes $P_{n}(x)$ de degrés égaux aux indices et les coefficients $A_{n}$, et on demande les courbes de convergence de ces séries. Ce problème inverse a été abordé, il y a 50 ans, par Darboux $\left({ }^{2}\right)$ et Ponncaré $\left({ }^{3}\right)$. Dans ce qui suit nous considérons les cas où les polynomes $P_{n}(x)$ sont donnés par des relations de récurrence de Poincaré pour lesquelles on trouve $\lim \frac{P_{n+1}}{P_{n}}$, ou quand on connait $\lim \sqrt[n]{P_{n}(x)}$. Nous construisons,

(1) FABER, Ueber polynomische Entwickelungen ("Math. Annalen ", Bd. 57, 1903, p. 389; Bd. 64, 1907, p. 118). Voir aussi, P. MoNTet, Leçons sur les séries de polynomes à une va. riable complexe.

(*) Darboux, Ménoire sur l'approximation des fonctions de très grands nombres et sur wne classe étendue de développements en séries ("Journal de Math. pures et appliquées", t. IV, 1878 , p. 411).

(3) Pormcaré, Sur les équations linéaires aux différentielles ordinaires et aux différences finies ("American Journal of Math.", vol. VII, 1884, p. 201). 
comme application, des fonctions holomorphes dans des domaines limités par de courbes donnèes.

Les séries $\Sigma \frac{A_{n}}{P_{n}(x)}$ suivant les inverses de polynomes donnés, rencontrées par MM. Gurluet et AubenT (6), ont été étudiées en premier lieu par M. APpenc (\%). Nous étudions les courbes de convergence dans les cas où les polynomes $P_{n}(x)$ sont donnés par de relations de récurrence de Poincaré, ou quand on connait $\lim \sqrt[n]{\vee} \overline{P_{n}(x)}$; de mème pour les séries $\Sigma A_{n} P_{n}(x)+-\Sigma \frac{B_{n}}{P_{n}(x)}\left({ }^{8}\right)$.

\section{Les séries $\Sigma A_{n} P_{n}(x)$.}

1. Les courbes de convergence des séries $\Sigma A_{n} P_{n}(x)$, les polynomes $P_{n}(x)$ étant liés par de relations de récurrence de Poincaré. Séries de Poincaré. Considérons la série

$$
\text { \ } A_{n} P_{1,}(x)
$$

les polynomes $P_{,}(x)$ étant liés par la relation de récurrence de Poincaré

$$
R_{k} P_{n+k}+R_{k-1} P_{n+k-1}+\ldots+R_{0} P_{n}=0
$$

$A_{0}, A_{1}, \ldots A_{n}, \ldots$ étant donnés tels que

$$
\overline{\lim } \sqrt[n]{\left|A_{n}\right|}=\frac{1}{l}
$$

et $R_{q}$ de fonctions qui dépendent de $x$ et de rang $n$. Ces séries ont été considérées premièrement par PoIncaré $\left({ }^{3}\right),\left({ }^{4}\right),\left({ }^{5}\right)$. On sait que le rapport $P_{n+1}: P_{n}$

(4) S. PrvoherLe, Sui sistemi di funetoni analitiche... (" Annali di Matematica «, II, vol. XII).

(5) E. PrCard, Cours d'Analyse supérieure de la Sorbonne, en 1912, 1918. Les sévies de Darboux et de Ponncarè on été considérées par M. Picard dans son Traité d'Analyse, t. IIT, p. 419 .

(6) Guillet et Aubert, *Comptes Rendus », t. 155, 1912, pp. 139, 204, 708, 820.

(1) P. Appext, Sur les développements en séries snivant les inverses de polynones donnés ("Comptes Rendus", t. 157, 1913, pp. 5̆, 1042); "Bulletin des Sciences Math. », $2^{\mathrm{e}}$ série, t. 37, 1913, p. 345; "Bulletin de la Societe Math. de France», t. 48, 1920, pp. 1-8).

${ }^{8}$ ) Voir mes Totes: Snlle serie di polinomi di ma variabile complessa. Le serie di Darboux ("Annali di Mat. pura ed applicata», serie IIT, t. XXXI, 1922, p. 207); Sur les séries de polynomes d̀ une variable complexe ("Journal de Math. pures et appliquées ", ge série, t. I, 1922, p. 77); Sur les courbes de convergence des séries procédant suivant les inverses de polynomes donmés ("Comptes Rendus ", t. 180, 1925, p. 566); Snlle relazioni ricorrenti di ordine infunito ("Bollettino dell'Unione Mat. Italiana », anno IV, n. 4, 1925, p. 155); Nowvelle méthode pour l'etude des régions de convergence des séries de polynomes 
tend, en général, vers la racine $p(x)$ de plus grand module de l'équation

$$
F(\lambda)=O_{k} \lambda^{k}+\ldots+C_{0}=0, \quad C_{s}=\lim _{n \rightarrow \infty} \frac{R_{s}}{R_{k}} .
$$

Pour trouver les courbes de convergence de ces séries, considérons la série

$$
A_{0}+A_{1} x+\ldots+A_{n} x^{n}+\ldots,
$$

dont le rayon de convergence est $l$. Supposons premièrement $|p(x)|<l$ et considérons un nombre $l_{1}$ tel que $|p|<l_{1}<l$. On a

$$
\lim \frac{P_{n+1}}{P_{n}}=p(x), \quad\left|\frac{P_{n+1}+1}{P_{n}}\right|<l_{1}<l,
$$

et si, pour fixer les idées, on suppose que l'on a cette inégalité en partant de $n=0$, nous aurons

$$
\begin{gathered}
\left|\frac{P_{1}}{P_{0}}\right|<l_{1},\left|\frac{P_{2}}{P_{1}}\right|<l_{1}, \ldots,\left|\frac{P_{n 2}}{P_{n-1}}\right|<l_{1}, \\
\left|P_{n}\right|<\left|P_{0}\right| l_{1}^{n}, \quad\left|A_{n} P_{n}\right|<\left|P_{0}\right| \cdot\left|A_{n} l_{1}^{n}\right| .
\end{gathered}
$$

Done, si $|p(x)|<l$, la série (1) est convergente, comme ayant les modules de ses termes plus petits que ceux de la sèrie convergente $\Sigma A_{n} l_{1}^{n}, l_{1}<l$.

Supposons, au contraire, que $|p(x)|>l$, et que $|p|>l_{2}>l$. Nous aurons

$$
\left|\frac{P_{n+1}+1}{P_{n}}\right|>l_{2}, \quad\left|P_{n}\right|>\left|P_{0}\right| l_{2}{ }^{n}, \quad\left|A_{n} P_{n}(x)\right|>\left|P_{0}\right| \cdot\left|A_{n} l_{1}^{n}\right| \text {. }
$$

Done, si $|p(x)|>l$, la série (1) est divergente, comme ayant les modules de ses termes plus grands que ceux d'une série divergente, $\Sigma A_{n_{1}} l_{2}{ }^{n}, l_{2}>l$.

Il en résulte que la courbe de convergence de la série de Poincaré, $\Sigma A_{n} P_{n}(x)$, où les polynomes $P_{n}(x)$ sont donnés par la relation de récurrence (2), est donnée par l'équation $|p(x)|=l, p(x)$ étant la racine de plus grand module de l'équation (3) et $\frac{1}{l}=\varlimsup \lim \sqrt[n]{\left|A_{n}\right|}$. La série est valable senlement à l'intérieur de la courbe $|p(x)|=l, x=X+i Y$.

2. Exemples. - $1^{\circ} P_{n}(x)$ les polynomes de Legendre

$$
P_{n}(x)=\frac{1}{2^{n} n !} \frac{d^{n}\left(x^{2}-1\right)^{n}}{d x^{n}}
$$

orthogonaux («Bulletin des Sciences Math. , t. LIV, nov. 1930); Sur le facten* de conver* gence uniforme de M. Leja d'une série de polynomes ("Comptes Rendus ", t. 193, 19:1, p. 984); Sur la determination de fonctions holomorphes dans des domaines donnés ("Comptes Ren* dus ", t. 194, 1932, p. 163); Les polynones onthogonanx ("Annales de Tonlouse », t. 24, 1982, pp. $67-87$ ). 
la relation (2) et l'équation (3) sont

$$
n P_{n}-(2 n+1) x P_{n-1}+(n-1) P_{n \rightarrow 2}=0, \quad \lambda^{2}-2 \lambda x+1=0 .
$$

La courbe de convergence de la série $\Sigma A_{n} P_{n}(x), \frac{1}{l}=\overline{\lim } \sqrt[n]{\left|A_{n}\right|}$, est donnée par $\mid p(x)\}=l, p(x)$ étant la racine de plus grand module de l'équation $\lambda^{2}-2 \lambda x+1=0$, qu' on peut ecrire

$$
x=\frac{1}{2}\left(\lambda+\frac{1}{\lambda}\right) .
$$

Donc, la courbe de convergence est donnée par

$$
x=\frac{1}{2}\left(\lambda+\frac{1}{\lambda}\right), \quad|\lambda|=l,
$$

et en posant $\lambda=l e^{i \theta}, x=X+i Y$, on a

$$
X=\frac{1}{2}\left(l+\frac{1}{l}\right) \cos \theta, \quad Y=\frac{1}{2}\left(l-\frac{1}{l}\right) \sin \theta,
$$

ce qui montre que cette courbe est l'ellipse

$$
\frac{4 X^{2}}{\left(l+\frac{1}{l}\right)^{2}}+\frac{4 Y^{2}}{\left(l-\frac{1}{l}\right)^{2}}=1
$$

de foyers -1 et +.1 , et la série est valable senlement à l'intérieur de cette ellipse.

$2^{\circ}$ Considérons la série $\mathrm{\Sigma} \mathrm{A}_{\mathrm{n}} \mathrm{P}_{\mathrm{n}}(\mathrm{x})$, les polynomes $\mathrm{P}_{\mathrm{n}}(\mathrm{x})$ étant donnés par

$$
\frac{d^{n} y}{d x^{n}}=\frac{P_{n}(x)}{\left(1+x^{2}\right)^{n+1}}, \quad y=\frac{1}{1+x^{2}}
$$

et qui vérifient la relation de récurrence

$$
P_{n}+2 n x P_{n-1}+n(n-1)\left(1+x^{2}\right) P_{n-2}=0
$$

Changeant $n$ en $n+2$, cette relation devient

$$
P_{n+2}+2(n+2) x P_{n+1}+(n+2)(n+1)\left(1+x^{2}\right) P_{n}=0 .
$$

Les coefficients $R_{q}$ de cette relation de récurrence n' étant pas de mème degré, posons $P_{n}=(n !) P^{\prime}{ }_{n}$ et l'on a $\left({ }^{9}\right)$

$$
\begin{gathered}
(n+2)(n+1) \mu P_{n+2}^{\prime}+2(n+2)(n+1) x P_{n+1}^{\prime}+ \\
+(n+2)(n+1)\left(1+x^{2}\right) P_{n}^{\prime}=0
\end{gathered}
$$

(9) Les conrbes de convergence des séries $\Sigma A_{n} P_{q i}^{\prime}$ sont les mêmes que celles des séries $\Sigma A_{n} P_{n}$. 
et la relation de récurrence générale (2) devient

$$
P_{n+k}^{\prime}+\frac{S_{k-1}}{(n+k)^{\mu}} P_{n+k-1}^{\prime}+\frac{S_{k-2}}{[(n+k)(n+k-1)]^{\mu}} P_{n+k-2}^{\prime}+\ldots=0 .
$$

Il faut déterminer $\mu$ tel que pour $n \rightarrow \infty$, les expressions

$$
\left[\frac{(n+i) !}{(n+k) !}\right]^{\mu} S_{i}
$$

soient finies. Dans notre cas, il suffit de prendre $\mu=1$, et la relation (4) devient

$$
P_{n+2}^{\prime}+2 x P_{n+1}^{\prime}+\left(1+x^{2}\right) P_{n}^{\prime}=0 .
$$

L' équation qui donne la limite da rapport $P^{\prime}{ }_{n+1}: P^{\prime}{ }_{n}$ est

d'où

$$
\lambda^{2}+2 \lambda x+1+x^{2}=0
$$

Si $x=X+i Y$, on a

$$
\lambda=-x \pm i \text {. }
$$

$$
\begin{gathered}
\lambda^{\prime}=-x+i=-X+i(1-Y) . \quad \lambda^{\prime \prime}=-x-i=-X-i(1+Y) \\
\left|\lambda^{\prime}\right|^{2}=X^{2}+(1-Y)^{2}, \quad\left|\lambda^{\prime \prime}\right|^{2}=X^{2}+(1+Y)^{2}, \quad\left|\lambda^{\prime \prime}\right|>\left|\lambda^{\prime}\right| .
\end{gathered}
$$

Les courbes de convergence sont les cercles

$$
\left|\lambda^{\prime \prime}\right|=l=\text { const. }, \quad X^{2}+(1+Y)^{2}=l^{2} .
$$

$3^{\circ}$ Considérons la série $\Sigma \mathrm{A}_{\mathrm{n}} \mathrm{P}_{\mathrm{n}}(\mathrm{x}), \mathrm{P}_{\mathrm{n}}(\mathrm{x})$ étant les polynomes de Laguerre, qui admettent la fonction génératrice

$$
e^{\frac{\not x x}{1-\alpha}}=(1-\alpha) \Sigma \alpha^{n} P_{n}(x)
$$

Prenant la dérivée par rapport à $\alpha$, on trouve

$$
\begin{aligned}
& \frac{e^{\frac{\alpha x}{1-\alpha}}}{1-\alpha} \frac{x}{1-\alpha}=(1-\alpha) \Sigma n \alpha^{n-1} P_{n}(x)-\Sigma \alpha^{n} P_{n}(x), \\
& \Sigma \alpha^{n} P_{n} \frac{x}{1-\alpha}=(1-\alpha) \Sigma n \alpha^{n-1} P_{n}-\Sigma \alpha^{n} P_{n}, \\
& x \Sigma \alpha^{n} P_{n}=(1-\alpha)^{2} \Sigma n \alpha^{n-1} P_{n}-(1-\alpha) \Sigma \alpha^{n} P_{n} .
\end{aligned}
$$

Égalant les coefficients de $\alpha^{n}$, on trouve la relation de récurrence

$$
(n+1) P_{n+1}-(x+2 n+1) P_{n}+n P_{n-1}=0 \text {. }
$$

Changeant $n$ en $n+1$, on a

$$
(n+2) P_{n+2}-(x+2 n+3) P_{n+1}+(n+1) P_{n}=0 .
$$


Pour $n \rightarrow \infty, \quad \lim \frac{P_{n+1}}{P_{n}}=\lambda, \quad F(\lambda)=\lambda^{2}-2 \lambda+1=0, \quad \lambda=1, \quad F(1)=0$, $\left(\frac{d F^{\prime}}{d \lambda}\right)_{\lambda=1}=0, \lim \frac{P_{n+1}}{P_{n z}}=1$, indépendante de $x$.

En désignant par $l$ le rayon de convergence de la série $\Sigma A_{n} x "$, si $l>1$ $\left[\lim \left|\frac{P_{n+1}}{P_{n}}\right|=\lambda=1\right]$ la série $\Sigma A_{n} P_{n}(x)$ est toujours convergente; si ce rayon $l=1$, le critérium fondé sur la limite du rapport $\frac{u_{n+1}}{u_{n}}$ est en défaut. On peut employer le. critère imaginé par Poincaré. En écrivant le rapport sous la forme

$$
\frac{u_{n+1}+1}{u_{n}}=1-\frac{\beta_{n}}{n}
$$

on cherche la limite $\beta$ de $\beta_{n}$ pour $n \rightarrow \infty$. Si cette limite a sa partie réelle plus grande que 1 , la série $\Sigma u_{\eta}$ est convergente, si cette partie réelle est plus petite que 1, la série est divergente.

Cela posé, trouvons les courbes de convergence des séries $\Sigma A_{n_{1}} P_{n}(x)$, pour lesquelles

$$
\lim \frac{P_{n+1}}{P_{n}}=1, \quad \lim \frac{A_{n+1}}{A_{n}}=1 .
$$

Posant

$$
\frac{P_{n+1}}{P_{n}}=1-\frac{\beta_{n}}{n}, \quad \lim \beta_{n}=\beta, \quad \frac{A_{n+1}}{A_{n}}=1-\frac{\gamma_{n}}{n}, \quad \lim \gamma_{n}=\gamma,
$$

on a

$$
\frac{A_{n+1}+1}{A_{n}} \frac{P_{n+1}}{P_{n}}=\left(1-\frac{\gamma_{n}}{n}\right)\left(1-\frac{\beta_{n}}{n}\right)=1-\frac{\beta_{n}+\gamma_{n}}{n}+\frac{\beta_{n} \gamma_{n}}{n^{2}}
$$

et en appliquant le critère de convergence employé, on trouve que la condition de convergence de la série $\Sigma A_{n} P_{n}$ est donnée par la relation: la partie réelle de $(\beta+\gamma)>1$. Comme seulement $\beta$ depend de $x$, les courbes de convergence des séries $\Sigma A_{n} P_{n}(x)$ sont données par: la partie réelle de $\beta=$ const.

Dans le cas des polynomes de Laguerre, pour lesquels $F(\lambda)=\lambda^{2}$. $-2 \lambda+1=0, F(1)=0,\left[\frac{d F}{d \lambda}\right]_{\lambda=i}=0$, il faut substituer

$$
P_{n+1}=P_{n}\left(1-\frac{\beta}{\sqrt{n}}-\frac{\delta_{n}}{n}\right), \quad P_{n+2}=P_{n}\left(1-\frac{\beta}{\sqrt{n+1}}-\frac{\delta_{n+1}}{n+1}\right)\left(1-\frac{\beta}{\sqrt{n}}-\frac{\delta_{n}}{n}\right),
$$

et la relation de récurrence (5) devient

$$
\begin{gathered}
(n+2)\left(1-\frac{\beta}{\sqrt{n+1}}-\frac{\delta_{n+1}}{n+1}\right)\left(1-\frac{\beta}{\sqrt{n}}-\frac{\delta_{n}}{n}\right)- \\
-(x+2 n+3)\left(1-\frac{\beta}{\sqrt{n}}-\frac{\delta_{n z}}{n}\right)+n+1=0, \\
\left(\beta^{2}-x\right)+\left(\delta_{n+1}-\delta_{n}\right)+H=0 .
\end{gathered}
$$


Si $\lim \delta_{n}=\delta$, la dernière parenthèse est nulle, donc, pour $n \rightarrow \infty$, il reste $\beta^{2}-x=0$, car $H$ est la somme des termes qui s'annulent avec $\frac{1}{n}$. Done $\beta^{2}=x$. Les courbes de convergence sont données par: la partie réelle de $\beta=$ const.

Posant $\beta=u+i v, x=X+i Y$, on a, de $\beta^{2}=x$,

$$
X=u^{2}-v^{2}, \quad Y=2 u v, \quad Y^{2}+4 u^{2} X-4 u^{4}=0,
$$

et comme $u$ la partie réelle de $\beta$ est une constante, il résulte que les courbes de convergence des séries de polynomes de Laguerre, $\Sigma A_{n} P_{n}(x)$, avec $\lim \frac{A_{n+1}}{A_{n}}=1$, sont les paraboles $Y^{2}+4 u^{2} X-4 u^{4}=0$, avec le foyer en origine.

3. Les courbes de convergence des séries $\mathbb{\Sigma} A_{n} P_{n}(x)$ pour lesquelles on connait $\varlimsup \sqrt[n]{\left|P_{n}(x)\right|}=|p(x)|, \varlimsup \lim \sqrt{\left|A_{n}\right|}=\frac{1}{l}$. - I) Supposons d'abord $|p(x)|<l$. On peut trouver $p_{1}$ et $l_{1}$, tels que

On a

$$
|p(x)|<p_{1}<l_{1}<l .
$$

$$
\left|A_{n}\right|<\frac{1}{l_{1}^{n}}, \quad\left|P_{n}(x)\right|<p_{1}^{n}, \quad\left|A_{n} P_{n}(x)\right|<\left(\frac{p_{1}}{l_{1}}\right)^{n}
$$

et done la série est valable, car ses termes sont en valeur absolue plus petits que les termes d'une série convergente. Supposons, an contraire, $|p(x)|>l$. On peut trouver $p_{2}$ et $l_{2}$, tels que

On a

$$
|p(x)|>p_{2}>l_{2}>l
$$

$$
\left|A_{n}\right|>\frac{1}{l_{2}{ }^{n}}, \quad\left|P_{n}(x)\right|>p_{2}{ }^{n}, \quad\left|A_{n} P_{n}(x)\right|>\left(\frac{p_{2}}{l_{2}}\right)^{n}
$$

et la série diverge.

Donc, la courbe de convergence de la série $\Sigma A_{n} P_{n}(x)$ est donnée par $|p(x)|=l$, et la série est valable dans la région intérieure à cette courbe.

II) Considérons le cas où les suites

$$
\sqrt[n]{\left|A_{n}\right|} \text { et } \sqrt[n]{\left|P_{n}(x)\right|}, \quad n=0,1,2, \ldots
$$

ne tendent pas vers de limites uniques pour $n \rightarrow \infty$, quand la région de convergence de la série $\Sigma A_{n} P_{n},(x)$ ne dependra seulement en général des limites supérieures des suites (6), mais aussi des autres points limites de 
ces mèmes suites, ce qu'on voit dans l'exemple suivant communiqué par M. Karamata. Considérons dans la série $S(x)=\underset{n=0}{\infty} A_{n} P_{n}(x)$, où les polynomes $P_{n}(x)$ sont de la forme

$$
P_{n}(x)=\left[x+(-1)^{n}\right]^{n}, \quad n=0,1,2, \ldots,
$$

séparément les sommes $S_{1}(x)$ des termes de rang pair et $S_{2}(x)$ des termes de rang impair. La série $S(x)$ convergera dans le domaine $(D)$ intérieur anx cercles

$$
\begin{aligned}
& |x+1|=l_{1}, \quad \limsup _{n \rightarrow \infty} \sqrt[2 n]{\left|A_{2 n}\right|}=\frac{1}{l_{1}^{-}} \\
& |x-1|=l_{2}, \quad \limsup _{n \rightarrow \infty} \sqrt[2 n+1]{\left|A_{2 n+1}\right|}=\frac{1}{l_{2}} .
\end{aligned}
$$

D'autre part,

$$
|\not(x)|=\limsup _{n \rightarrow \infty} \sqrt[n]{\left|P_{2 \ell}(x)\right|}=\limsup _{n \rightarrow \infty}\left|x+(-1)^{n}\right|
$$

done le domaine $\left(D^{\prime}\right)|p(x)|<l$, où $\frac{1}{l}=\limsup _{n \rightarrow \infty} \sqrt[n]{\left|A_{n}\right|}$, est la partie com. mune aux deux cercles

$$
|x+1|=l, \quad|x-1|=l,
$$

et puisque, dans le cas géneral, $l<l_{1}, l<l_{2}$, le domaine $\left(D^{\prime}\right)$ sera contenu dans le domaine $(D)$. Les denx cercles $|x+1|=l,|x-1|=l$ se coupent si leur rayon $l>1$, on ce qui revient an mème si la série $\Sigma A_{n}$ est convergente.

Ainsi que, dans le cas général, du fait que

$$
\limsup _{n \rightarrow \infty} \sqrt[n]{\mid A_{n} P_{n}(x)}\left|\leqq \limsup _{n \rightarrow \infty} \sqrt[n]{\left|A_{n}\right|} \cdot \limsup _{n \rightarrow \infty} \sqrt[n]{\left|P_{n}(x)\right|}=\frac{1}{l}\right| p(x) \mid
$$

la série $S(x)$ convergera toujours à l'intérieur du domaine $(D)$ défini par $|p(x)|=l$, mais $|p(x)|=l$ ne sera pas en général la courbe limitant le domaine de convergence de la série $S(x)$. Cependant, si l'une des deux suites (6) tendent vers une limite déterminée pour $n \rightarrow \infty$, la série $S(x)$ converge seulement à l'intérieur de cette courbe.

Des exemples de séries de polynomes offrant de singularités de convergence plus profondes ont été données par M. HELGE voN KooH (io).

(10) HELGE voN KocH, Renarques sur quelques séries de polynomes («Bulletin de la Société Math. de France», t. XXXIV, 1906, pp. 269-274). 
4. Les séries de polynomes orthogonaux. - I) Supposons que $P_{n}(x)$ soient les polynomes orthogonaux, donnés par

$$
\int_{a}^{b} \varphi(x) P_{n n}(x) P_{n}(x) d x=0, \quad m \leqq n, \quad \int_{a}^{b} \varphi(x) P_{n}{ }^{2}(x) d x=I_{n}==\text { const. }
$$

On sait ( $\left.{ }^{8}\right)$ que

$$
\varlimsup \sqrt[n]{\lim } \overline{\left|P_{n}(x)\right|}=\frac{b-a}{4}|z|
$$

z étant la racine de plus grand module de l'équation

$$
z^{2}-\frac{4}{b-a}\left(x-\frac{a+b}{2}\right) z+1=0
$$

Les courbes de convergence des séries $\Sigma A_{n} P_{n}(x), \frac{1}{l}=\varlimsup_{\lim } \sqrt[n]{\left|A_{n}\right|}$, sont données par $\frac{b-a}{4}|z|=l$, avec la relation (7), on par

$$
x=\frac{a+b}{2}+\frac{b-a}{4}\left(z+\frac{1}{z}\right), \quad|z|=l \frac{4}{b-a},
$$

et sont des ellipses de foyers $a$ et $b$.

II) Remarque relative ’̀ la relation de récurrence entre trois polynomes orthogonaux consécutifs. On sait $\left({ }^{8}\right)$ que les polynomes orthogonaux

$$
P_{n}(x)=c_{n, n} x^{n}+c_{n, n-1} x^{n-1}+\ldots+c_{n, 0}, \quad c_{n, n}=1,
$$

vérifient la relation de récurrence

$$
\begin{aligned}
& P_{n+1}(x)-\left(x-u_{n}\right) P_{z}(x)+v_{n} P_{n-1}(x)=0,
\end{aligned}
$$

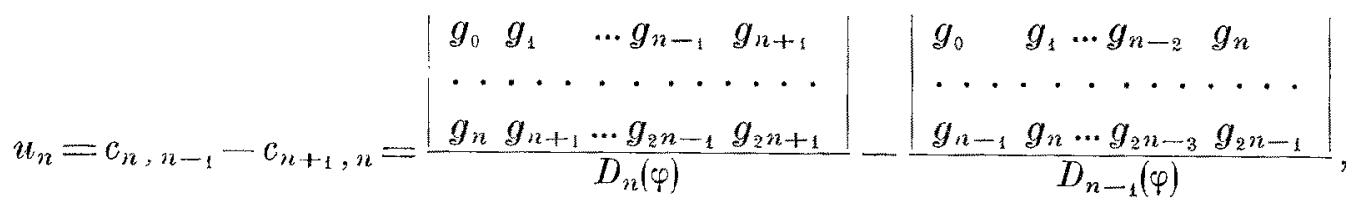

$$
\begin{aligned}
& v_{n}=\frac{I_{n}}{I_{n-1}}, \quad I_{n}=\frac{D_{n}(\varphi)}{D_{n-1}(\varphi)}, \quad D_{n}(\varphi)=\left|\begin{array}{cccc}
g_{0} & g_{1} & \ldots & g_{n} \\
g_{1} & g_{2} & \ldots & g_{n+1} \\
\ldots & \ldots & \ldots & \ldots \\
g_{n} & g_{n+1} & \ldots & g_{2 n}
\end{array}\right|, \quad g_{s}=\int_{a}^{b} \varphi(t) t^{s} d t
\end{aligned}
$$

Le développement $\Sigma A_{n} P_{n}(x)$ étant valable dans la région de convergence trouvée, et $\lim \frac{P_{n z}+1}{P_{n}}=\lambda$ étant la racine de l'équation

$$
\lambda^{2}-(x-u) \lambda+v=0
$$


qu' on obtient $\left(n^{\circ} 1\right)$ de $(8)$, il suit $q$ ue $\lim \left|\frac{P_{n+1}}{P_{n}}\right|=\lim \sqrt[n]{\mid P_{n}} \mid$, et done $\lambda=\frac{b-a}{4}|z|$, étant la racine de I'equation (7); done $\lim \frac{P_{n+1}}{P_{n}}=\lambda$ est la racine de l'équation

$$
\lambda^{2}-\left(x-\frac{a+b}{2}\right) \lambda+\left(\frac{b-a}{4}\right)^{2}=0
$$

et en comparant avee (9), on trouve

$$
u=\lim u_{n}=\frac{a+b}{2}, \quad v=\lim v_{n}=\left(\frac{b-a}{4}\right)^{2} .
$$

III) Comme cas particuliers des polynomes orthogonaux sont les poly. nomes de Legendre, pour lesquelśs $\varphi(x)=1, a=-1, b=1$, les polynomes de Jacobi, où

$$
\varphi(x)=(x-a)^{2}(x-b)^{\mu}, \quad \lambda+1>0, \quad \mu+1>v
$$

les polynomes qui résultent de la série hypergéométrique, considérés par Darboux $\left({ }^{2}\right)$,

$$
\varphi(x)=x^{\gamma-1}(1-x)^{\alpha-\gamma}, \quad \gamma>0, \quad \alpha-\gamma+1>0, \quad a=0, \quad b=1 .
$$

Un cas intéressant des polynomes orthogonaux ést donné par les polymomes de Laguerre, qui peuvent ètre obtenus de la manière suivante. En posant

considérons l'intégrale

$$
P_{n}(x)=a_{0}+a_{1} x+\ldots+a_{n} x^{n}
$$

$$
I(y)=\int_{0}^{\infty} e^{-x} x^{y-1} P_{n}(x) d x=\sum_{i=0}^{n} a_{i} \int_{0}^{\infty} e^{-x} x^{y+i-1} d x .
$$

On sait que

Done

$$
\Gamma(a)=\int_{0}^{\infty} e^{-x} x^{a-1} d x, \quad \Gamma(a+n)=a(a+1) \ldots(a+n-1) \Gamma(a) .
$$

$$
\begin{gathered}
I(y)=\sum a_{i} \Gamma(y+i), \\
I(y)=\Gamma(y) \sum_{i=0}^{n} \alpha_{i} y(y+1) \ldots(y+i-1) .
\end{gathered}
$$

L'intégrale $I(y)$ est nulle quand

on

$$
\sum_{i=0}^{n} \alpha_{i} y(y+1) \ldots(y+i-1)=0
$$

(11) $Q(y)=a_{n} y(y+1) \ldots(y+n-1)+a_{n-1} y(y+1) \ldots(y+n-2)+\ldots+a_{0}=0$. 
Écrivons que les polynomes $P_{n}(x)$ sont orthogonaux, e'est-à-dire

$$
\int_{0}^{\infty} e^{-x} x^{k} P_{n}(x) d x=0, \quad k=0,1,2, \ldots n-1 .
$$

Ces conditions expriment que l'intégrale (10) $I(y)=0$ poux $y=1,2, \ldots n$, et comme $I(y)=0$ est donnée par la relation (11), il suit que l'équation (11) a pour racines $1,2, \ldots n$. Donc, les coefficients $a_{0}, a_{1}, \ldots a_{n}$ se trouvent en écrivant que l'equation (11) a pour racines $1,2, \ldots n$, o' est-à-dire qu' elle est de la forme

$$
\begin{gathered}
(y-1)(y-2) \ldots(y-n)=a_{n} y(y+1) \ldots(y+n-1)+ \\
+a_{n-1} y(y+1) \ldots(y+n-2)+\ldots+a_{0} .
\end{gathered}
$$

Pour trouver ces coefficients, considérons les identités (la première est la dérivée avec la formule de Leibniz)

$$
\begin{gathered}
\frac{d^{n}\left(u^{n} u^{-y}\right)}{d u^{n}}=(-y)(-y-1) \ldots(-y-n+1) u^{-y}+ \\
+n C_{n}^{1}(-y)(-y-1) \ldots(-y-n+2) u^{-y}+\ldots+n ! u^{-y} \\
\frac{d^{n}\left(u^{n-y}\right)}{d u^{n}}=(n-y)(n-y-1) \ldots(-y+1) u^{-y}
\end{gathered}
$$

En identifiant ces expressions multipliées par $(-1)^{n}$ et en simplifiant avec $u^{-y}$, on a

$$
\begin{gathered}
(y-1)(y-2) \ldots(y-n)=y(y+1) \ldots(y+n-1)- \\
-n C_{n}{ }^{1} y(y+1) \ldots(y+n-2)+\ldots+(-1)^{n} n !
\end{gathered}
$$

En comparant avec (12), il suit

$$
a_{n-i}=(-1)^{n+i} n(n-1) \ldots(n-i+1) C_{n}{ }^{i},
$$

de sorte que l'expression des polynomes de Laguerre esç

$$
P_{n}(x)=(-1)^{n}\left[x^{n}-n C_{n}{ }^{1} x^{n-1}+n(n-1) C_{n}{ }^{2} x^{n-2}-\ldots+(-1)^{n} n !\right]=e^{x} \frac{d^{n}}{d x^{n}}\left(x^{n} e^{-x}\right)
$$

et satisfait à l'équation différentielle $x y^{\prime \prime}+(1-x) y^{\prime}+n y=0$.

Ces polynomes, utilisés dans la théorie de l'atome d'hydrogène dans la Mécanique ondulatoire, étant orthogonaux, donnés par

$$
\int_{a}^{b} \varphi(x) P_{m}(x) P_{n}(x) d x=0, m \lessgtr n ; \varphi(x)=e^{-x}, a=0, b=\infty, \int_{0}^{\infty} e^{-x} P_{n}{ }^{2}(x) d x=(n !)^{2},
$$

on sait que les courbes de convergence des séries de polynomes orthogonaux sont des ellipses de foyers $a$ et $b$, qui dans le cas des polynomes de Laguerre $(b \rightarrow \infty)$ sont des paraboles avec le foyer à l'origine $(a=0)$, de sorte que nous retrouvons le résultat obtenu $\left(n^{\circ} 2,3^{\circ}\right)$. 
ó. Détermination des fonctions holomorphes dans des domaines limités par des courbes données. -- On represente une fonction holomorphe dans un domaine $(D)$ par une série entiere, $f(z)=\Sigma a_{n} z^{\eta n}$, sans donner une expression d'où l'on pourrait voir que cette fonction est valable seulement dans ce domaine. Dans ce qui suit, nous construisons une fonction holomorphe représentée par une série de polynomes, qui est valable seulement à l'intérieur d'un contour (D) limité par une courbe (C).

Soit

$$
z=\frac{a}{Z}+\varphi(Z), \quad \varphi(Z)=a_{1} Z+a_{2} Z^{2}+\cdots+a_{n} Z^{n}+\cdots
$$

la transformation conforme qui fait correspondre le domaine extérieur à la courbe donnée $(O)$ au domaine intérieur du cercle de rayon $|\boldsymbol{Z}|=r$, la fonction $\varphi(Z)$ étant holomorphe à l'intérieur du cercle de rayou $r$, donné par $\frac{1}{r}=\overline{\lim } \sqrt[n]{\left|a_{n}\right|}$. Considérons les polynomes $P_{n}(x)$ donnés par les relations de récurrence d'ordre infini

$$
\begin{gathered}
a P_{n+1}=x P_{n}-a_{1} P_{n-1}-\ldots-a_{n-1} P_{1}+(n+1) a_{n}, \\
P_{0}(x)=1, \quad P_{1}(x)=-\frac{x}{a}, \quad a P_{2}-x P_{2}=2 a_{1}, \ldots
\end{gathered}
$$

Il résulte que $x$ appartenant à une certaine région de convergence, la série on $Z$,

$$
P_{1}+Z P_{2}+\ldots+Z^{n} P_{n+1}+\ldots,
$$

est le quotient des deux séries

$$
\frac{c_{1}+c_{2} Z+\ldots+e_{n+1} Z^{n}+\ldots}{b_{1}+b_{2} Z+\ldots+b_{n+1} Z^{n}+\ldots}=P_{1}+Z P_{2}+\ldots+Z^{n} P_{n+1}+\ldots, \quad b_{1} \lessgtr 0 .
$$

D'où, par identification, les relations trouvées

$$
P_{1} b_{1}=c_{1}, \quad P_{2} b_{1}+P_{1} b_{2}=c_{z}, \quad P_{3} b_{1}+P_{2} b_{2}+P_{1} b_{3}=c_{3}, \cdots
$$

doivent ètre les relations (14). Done

$$
\begin{gathered}
c_{1}=-x, \quad c_{2}=2 a_{1}, \quad c_{3}=3 a_{2}, \ldots c_{n+1}=(n+1) a_{n}, \ldots \\
b_{1}=a, \quad b_{2}=-x, \quad b_{3}=a_{1}, \ldots b_{n+1}=a_{n-1}, \ldots
\end{gathered}
$$

et les séries $\Sigma c_{n} Z^{n}, \Sigma b_{n} Z^{n}$ ont le mème cercle de convergence de rayon $r$, et done la série (15), qui est le quotient des ces denx séries, a un rayon de convergence $|Z| \leqq r$.

Remplaçant en (16) les valeurs de $c_{s}$ et $b_{s}$, on a

$$
\frac{Z \varphi^{\prime}(Z)+\varphi(Z)-x}{a+Z \varphi(Z)-Z}=P_{1}+\grave{Z} P_{2}+\ldots
$$


ou

$$
\frac{-\frac{a}{Z^{2}}+\varphi^{\prime}(Z)}{\frac{a}{Z}+\varphi(Z)-x}=-\frac{1}{Z}+P_{1}(x)+Z P_{2}(x)+\ldots
$$

En posant

$$
z=\frac{a}{Z}+\varphi(Z)
$$

nous aurons le développement

$$
\frac{z^{\prime}}{z-x}=-\frac{1}{Z}+P_{1}(x)+Z P_{2}(x)+\ldots
$$

Or, la série $P_{1}(x)+Z P_{2}(x)+\ldots$, ou $\Sigma Z^{n} P_{n}(x)$, a pour rayon de con* vergence $|Z|=\frac{1}{\overline{\lim } \sqrt[n]{\left|P_{n}(x)\right|}}$, et le développement (19) est valable seulement pour $|x|<\mid z !$, c'est-à-dire pour les points $x$ intérieurs a la courbe $(O)$ obtenue par la transformation conforme (13)

$$
z=\frac{a}{Z}+\varphi(Z)
$$

Done

$$
\lim _{n \rightarrow \infty} \sqrt[n]{\left|P_{n}(x)\right|}=\frac{1}{Z}, \quad x=\frac{\alpha}{Z}+\varphi(Z), \quad \varphi(Z)=a_{1} Z+a_{2} Z^{2}+\ldots
$$

Considérons une série de polynomes, $\Sigma A_{n} P_{n}(x)$, les polynomes $P_{n}(x)$ étant donnés par les relations (14), ou par la fonction génératrice (17) ou (18), et les coefficients $A_{n}$ tels $q u e \frac{1}{r}=\overline{\lim } \sqrt[n]{\left|A_{n}\right|}$. On sait (no 3$)$ que la courbe de convergence de cette série est donnée par $|p(x)|=r$, ou

$$
x=\frac{a}{Z}+\varphi(Z), \frac{1}{|Z|}=r
$$

c'est-à-dire la courbe donnée $(C)$, et la série est valable seulement à l'interieur de cefte courbe. Donc la série $\Sigma A_{n} P_{n}(x)$ représente une fonction holomorphe seulement à l'intérieur de la courbe $(C)$.

ExEMPLEs. - $1^{\circ}$ Considérons l'ellipse

$$
x=\frac{1}{2}\left(Z+\frac{1}{Z}\right), \quad|Z|=\frac{1}{l}
$$

On a $x=\frac{a}{Z}+\varphi(Z), a=\frac{1}{2}, \varphi(Z)=\frac{1}{2} Z$, et donc la fonction génératrice (17), 
pour les polynomes attachés à cette trausformation, est

$$
\begin{gathered}
\frac{Z \varphi^{\prime}(Z)+\varphi(Z)-x}{a+Z \varphi(Z)-Z x}=-\Sigma Z^{n} P_{n+1} \\
\frac{2 Z-2 x}{1-2 x Z+Z^{2}}=\frac{1}{Z-\left(x+\sqrt{x^{2}-1}\right)}+\frac{1}{Z-\left(x-\sqrt{x^{2}-1}\right)}= \\
=-\sum_{n=0}^{\infty} \frac{1}{\left(x+\sqrt{x^{2}-1}\right)^{n+1}}-\sum_{n=0}^{\infty} \frac{1}{\left(x-\sqrt{x^{2}-1}\right)^{n+1}} .
\end{gathered}
$$

On a

$$
\begin{aligned}
-P_{n}(x) & =\frac{1}{\left(x+\sqrt{x^{2}-1}\right)^{n}}+\frac{1}{\left(x-\sqrt{x^{2}-1}\right)^{n}} \\
& =\left(x-\sqrt{x^{2}-1}\right)^{n}+\left(x+\sqrt{x^{2}-1}\right)^{n}
\end{aligned}
$$

les polynomes de Tohebischef. La fonction $f(x)=\Sigma A_{n} P_{n}(x)$ est holomorphe seulement à l'intérieur de cette ellipse $\left(\frac{1}{l}=\overline{\lim } \sqrt[n]{\left|A_{n}\right|}\right)$.

$2^{\circ}$ Considérons les ovales de Cassini

$$
x=\frac{\sqrt{1+Z^{2}}}{Z}, \quad|Z|=\frac{1}{l} ; \quad \sqrt{|x-1||x+1|}=\frac{1}{|Z|}=l,
$$

pour les quelles on a

$$
x=\frac{a}{Z}+\varphi(Z)=\frac{\sqrt{1+Z^{2}}}{Z}, \quad a=1, \quad \varphi(Z)=\frac{1}{Z}\left(\sqrt{1+Z^{2}}-1\right)
$$

et done avec la relation (17) on peut former la fonction génératrice des polynomes $P_{n}(x)$,

$$
\begin{gathered}
\frac{\frac{Z}{\sqrt{1+Z^{2}}}-x}{\sqrt{1+Z^{2}}-x Z}=P_{1}+Z P_{2}+\ldots= \\
=\frac{-x+Z-\frac{Z^{3}}{2}+\frac{1 \cdot 3}{1 \cdot 2} \frac{Z^{5}}{2^{2}}-\frac{1 \cdot 3 \cdot 5}{1 \cdot 2 \cdot 3} \frac{Z^{7}}{2^{3}}+\ldots}{1-x Z+\frac{Z^{2}}{2}-\frac{1 \cdot 1}{1 \cdot 2} \frac{Z^{4}}{2^{2}}+\frac{1 \cdot 1 \cdot 3}{1 \cdot 2 \cdot 3} \frac{Z^{6}}{2^{3}}-\ldots} .
\end{gathered}
$$

Identifiant, on tronve

$$
\begin{aligned}
& P_{1}=-x \\
& P_{2}-x P_{1}=1 \\
& P_{3}-x P_{2}+\frac{1}{2} P_{1}=0 \\
& \ldots \ldots \ldots
\end{aligned}
$$


et de mème les coefficients $a_{n}$ de

$$
p(Z)=\frac{1}{2}\left(\sqrt{1+Z^{2}}-1\right)=\frac{Z}{2}-\frac{1 \cdot 1}{1 \cdot 2} Z^{3}+\frac{1 \cdot 1 \cdot 3 Z^{5}}{1 \cdot 2 \cdot 3}-\ldots
$$

on peut done former une fonction, $f(x)=\Sigma A_{n} P_{n}(x)$, holomorpe senlement à l'intérieur de cette courbe (11).

En général, la fonction $f(x)=\Sigma A_{n} P_{n}(x), \frac{1}{l}=\overline{\lim } \sqrt[n]{\left|A_{n}\right|}$, est holomorphe à l'intérieur du domaine limité par la courbe

quand on connait

$$
|p(x)|=l, \quad x=\varphi(Z), \quad|Z|=\text { const. },
$$

$$
\varlimsup \sqrt[n]{\left|P_{n}(x)\right|}=|p(x)|, \quad \text { ou } \quad \lim \left|\frac{P_{n+1}}{P_{n}}\right|=|p(x)|,
$$

comme nous avons vu dans le cas des polynomes orthogonaux ( $\mathrm{n}^{\circ} 4$ ), la courbe (20) étant une ellipse, ou pour les polynomes de Laguerre $\left(\mathrm{n}^{\circ} 2,3^{\circ}\right)$, la courbe (20) étant une parabole.

\section{Les séries $\Sigma \frac{A_{n}}{P_{n}(x)}$ de M. Appell.}

6. Les courbes de convergence des séries $\Sigma \frac{A_{n}}{P_{n}(x)}$, les polynomes $P_{n}(x)$ étant donnés par de relations de récurrence. - Considérons les polynomes $P_{n}(x)$ donnés par la relation de récurrence

$$
R_{k} P_{n+k}(x)+R_{k-1} P_{n+k-1}(x)+\ldots+R_{0} P_{n}(x)=0,
$$

$k$ étant un nombre donné et $R_{s}$ de fonctions données qui dependent de $x$ et dn rang $n$. On sait que le rapport $\frac{P_{n+1}}{P_{32}}$ tend, en général, vers la racine $p(x)$ de plus grand module de l'équation

$$
\lambda^{k}+C_{k-1} \lambda^{k-4}+\ldots+C_{0}=0, \quad C_{s}=\lim _{n \rightarrow \infty} \frac{R_{s}}{R_{k}} .
$$

(11) On pent encore obtenir un développenent $\Sigma\left(\alpha_{28} x+\beta_{\imath}\right)[(x-a)(x-b)]^{n}$ à l'intévieur d' une ovale de CAssini. Voir A. KIENAST, Ueber die Dar'stellung der analytischen Funtionen dureh Reihen die nach Potenzen eines Polynoms... ("Inaugural Dissertation, Universität Zürich", 1906). Voir aussi ma Note, Sur le developpenent d'une fonction suivant les puis. sances d'un polynome donné (Congrès des math. roumains à T. Severin, 8 Mai 1932). 
Pour trouver la région de convergence d'une série $\Sigma \frac{A_{n}}{P_{n}(x)}$ suivant les inverses de polynomes donnés de M. Appell, pour laquelle $\varlimsup_{\lim } \sqrt[n]{\left|A_{a l}\right|}=l$, supposons premièrement $|p(x)|>l$, et considérons un nombre $l_{1}$, tel que $l<l_{1}<|p|$. Nous avons

$$
\lim _{n \rightarrow \infty} \frac{P_{n+1}}{P_{n}}=p(x), \quad\left|\frac{P_{n+1}}{P_{n}}\right|>l_{1}>l
$$

et si, pour fixer les idées, nous supposons que l'on ait cette inégalité à partir de $n=0$, nous aurons

$$
\begin{aligned}
& \left|\frac{P_{1}}{P_{0}}\right|>l_{1}, \quad\left|\frac{P_{2}}{P_{1}}\right|>l_{1}, \ldots\left|\frac{P_{n}}{P_{n-1}-1}\right|>l_{1} ; \\
& \left|P_{n}\right|>\left|P_{0}\right| l_{1}^{n}, \quad\left|\frac{A_{n 2}}{P_{n}}\right|<\frac{1}{\left|P_{0}\right|} \mid \frac{A_{n} \mid}{l_{1}^{n}} .
\end{aligned}
$$

Done, si $|p(x)|>l$, la séxie de M. Appell est valable, comme ayant les modules de ses termes plus petits que ceux de la série convergente $\Sigma \frac{A_{n n}}{z^{n}}$, à l'extérieur de cercle $|z|=l, l_{\mathbf{1}}>l$.

Supposons, au contraire, que $|p(x)|<l$ et que $|p|<l_{2}<l$. Nous aurons

$$
\left|\frac{P_{n+1}+1}{P_{n}}\right|<l_{2}<l, \quad\left|P_{n}\right|<\left|P_{0}\right| l_{2}{ }^{n}, \quad\left|\frac{A_{n}}{P_{n n}}\right|>\frac{1}{\left|P_{0}\right|} \mid \frac{A_{n b} \mid}{l_{2}^{n}} .
$$

Donc, si $|p(x)|<l$, la série de M. Appell est divergente, comme ayant les modules de ses termes plus grands que ceux d'une série divergente $\Sigma \frac{A_{3 i}}{l_{2^{n}}}$, $l_{2}<l$.

Il en résulte que la courbe de convergence de la série de M. Appell, où les polynomes $P_{u}(x)$ sont liés par la relation de récurrence (20), est donnée par l'équation $|p(x)|=l, p(x)$ étant la racine de plus grand module de l'équation (22). La courbe $|p(x)|=l,(x=X+i Y)$, du plan $X O Y$, sépare le plan en deux régions, l'une intérieure à la courbe, l'autre extérieure où se trouvent les points à l'infini.

Pour une de ces régions, on a $|p(x)|-l>0$, pour l'autre $|p(x)|-l<0$; dono, d'après ce que nous avons vu, dans la premiére région, $|p(x)|-l>0$, la série de M. Appell converge, dans l'autre diverge. Or, ces séries convergent pour $x$ tendant vers l'infini, qui est dans la région extérieure à la courbe, et done la série sera convergente dans la région extérienre à la courbe $|p(x)|=l$, qui est en même temps extérieure à la courbe où se trouvent les racines des polynomes $P_{n}(x)$. 
Exemples. - $1^{\circ}$ Considérons les polynomes électrosphériques $P_{n}(x)$ qui admettent la fonction génératrice $\left({ }^{\circ}\right)$

$$
\frac{1}{1-2 t x+t^{2}}= \pm t^{n} P_{u}(x)
$$

Ces polynomes ont été rencontrés par MM. Guillet et Aubert dans leurs recherches sur l'attraction mutuelle des deux sphères électrisées, ou d'une sphère et d'un plan; la capacité commune des denx armateurs en présence est donnée, à un facteur près, par la série de M. Appell $\Sigma \frac{1}{P_{n}(x)}$. La relation de récurrence entre cos polynomes est

$$
P_{n+1}-2 x P_{n}+P_{n-1}=0
$$

et on voit, de cette relation, que la suite de Sturm: $P_{0}(x), P_{1}(x), \ldots P_{n}(x)$. devient pour -1 et 1

$$
1, \quad 2,3, \ldots,(-1)^{n}(n+1) ; 1,2,3, \ldots,(n+1)
$$

done la suite perd, entre -1 et $1, n$ variations, ef donc les polynomes $P_{n}(x)$ ont leurs racines réelles sur le segment $(-1,+1)$. L'équation (22) est $\lambda^{2}-2 \lambda x+1=0$, et la région de convergence de la série $\Sigma \frac{1}{P_{n}(x)}$, où $\lim \sqrt[n]{\left|A_{n}\right|}=1$, est limité par le segment $(-1,+1), x=\frac{1}{2}\left(Z+\frac{1}{Z}\right),|Z|=1$, et done cette série est valable dans tont le plan $X O Y(x=X+i Y)$, sauf la coupure $(-1,+1)$.

$2^{\circ}$ Supposons que $P_{n}(x)$ soient les polynomes de Legendre

$$
P_{n}(x)=\frac{1}{2^{n} n !} \frac{d^{n}\left(x^{2}-1\right)^{n}}{d x^{n}}
$$

qui ont toutes leurs racines réelles sur le segment $(-1,+1)$. La courbe de convergence de la série de II. Appell, $\Sigma \frac{A_{n}}{P_{z b}(x)}$, est l'ellipse

$$
x=\frac{1}{2}\left(Z+\frac{1}{Z}\right), \quad|Z|=l,
$$

et la région de convergence est le domaine extérieur à cette ellipse.

7. Les courbes de convergence des séries $\Sigma \frac{A_{n 2}}{P_{n}(x)}$, pour lesquelles $\lim \sqrt[n]{\left|P_{n}(x)\right|}=|p(x)|, \varlimsup \sqrt[n]{\left|A_{n}\right|}=l$. - Supposons d'abord $|p(x)|>l$. On 
peut trouver $l_{1}$ et $p_{1}$, tels que $l<l_{1}<p_{1}<|p(x)|$. On a

$$
\left|A_{n}\right|<l_{1}{ }^{n}, \quad\left|P_{n}(x)\right|>p_{1}{ }^{n}, \quad\left|\frac{A_{n}}{P_{n}\left(x_{1}\right)}\right|<\left(\frac{l_{1}}{p_{1}}\right)^{n},
$$

donc la série est valable, car ses termes sont en module plus petits que ceux d'une série convergente. Supposons an contraire, $|p(x)|<l$. On peut trouver $l_{2}$ et $p_{2}$, tels que $|p(x)|<p_{2}<l_{z}<l$. On a

$$
\left|A_{n}\right|>l_{2}{ }^{n}, \quad\left|P_{n}(x)\right|<p_{2}{ }^{n}, \quad\left|\frac{A_{n}}{P_{n}}\right|>\left(\frac{l_{2}}{p_{2}}\right)^{n},
$$

et la série de M. Appell diverge. Donc, la courbe de convergence de cette série est donnée par $|p(x)|=l$, et la région où la série est valable est extérieure à cette courbe et extérieure-à la courbe où sont les racines des polynomes $P_{n}(x)$.

Exempte. - $P_{n}(x)$ étant les polynomes orthogonaux, on sait $\left(\mathrm{n}^{\circ} 4\right)$ que

$$
\lim \sqrt[n]{\left|P_{n}(x)\right|}=\frac{b-a}{4}|z|
$$

z étant la racine de plus grand module de l'équation

$$
z^{2}-\frac{b-a}{t}\left(x-\frac{a+b}{2}\right) z+1=0
$$

La courbe de convergence de la série de M. Appell, $\Sigma \frac{A_{n q}}{\bar{P}_{n}(x)}$, est l'ellipse

$$
x=\frac{a+b}{2}+\frac{b-a}{4}\left(z+\frac{1}{z}\right), \quad|z|=\frac{4}{b-a} l,
$$

et la série est valable dans la région extérieure à cette courbe et extérieure à la courbe où sont les racines des polynomes $P_{n}(x)$, le segment $a b$.

8. La région de convergence des séries $\Sigma A_{n} P_{n}(x)+\Sigma \frac{B_{n}}{P_{n}(x)} \cdot$ - Supposons que $\overline{\lim } \sqrt[n]{\left|A_{n}\right|}=\frac{1}{l}, \varlimsup \lim \sqrt[n]{\left|B_{n}\right|}=l$, et que l'on connait, soit $\lim \frac{P_{n+1}}{P_{n}}=p(x)$, on $\overline{\lim } \sqrt[n]{\left|P_{n}(x)\right|}=|p(x)|$, et soient $(\Gamma)$ et $\left(\mathrm{I}^{\prime}\right)$ les courbes $|p(x)|=l,|p(x)|=l^{\prime}$, La série considérée est valable dans la région intérieure à la courbe $(\Gamma)$, extérieure à la courbe $\left(\Gamma^{\prime}\right)$ et extérieure à la courbe $(C)$ où sont les racines des polynomes $P_{n}(x)$.

Dans le cas des polynomes orthogonaux, le domaine de convergence de la série est une couronne formée par deux ellipses homofocales. 
Appltcation. - Soient $P_{n}(x)$ les polynomes attachés à la transformation $\left(n^{0} 5\right)$

$$
z=\frac{a}{Z}+\varphi(Z), \quad \varphi(Z)=a_{1} Z+\ldots+a_{n} Z^{n}+\ldots
$$

et $(\mathrm{I}),\left(\Gamma^{\prime}\right)$ les courbes correspondant aux valeurs

$$
\begin{gathered}
|Z|=\frac{1}{l}, \quad|Z|=\frac{1}{l^{\prime}} \\
\varlimsup \sqrt[n]{\mid \overline{A_{n} \mid}}=\frac{1}{l}, \quad \varlimsup_{\lim } \sqrt[n]{\left|B_{n}\right|}=l^{\prime}, \quad \lim \sqrt[n]{\left|a_{n}\right|}=\frac{1}{r} .
\end{gathered}
$$

La fonction

$$
f(x)=\Sigma A_{n z} P_{n}(x)+\Sigma \frac{B_{n}}{P_{n}(x)}
$$

est holomorphe dans la région intérieure à la courbe $(\Gamma)$, extérieure à la courbe $\left(\Gamma^{\prime}\right)$ et extérieure à la courbe $(c)$ où sont les racines des poly. nomes $P_{n}(x)$. 